\title{
Structure and thermodynamics of a ferrofluid monolayer
}

\author{
E. Lomba \\ Instituto de Química Física Rocasolano, CSIC, Serrano 119, E-28006 Madrid, Spain \\ F. Lado \\ Department of Physics, North Carolina State University, Raleigh, North Carolina 27695-8202 \\ J. J. Weis \\ Laboratoire de Physique Théorique, Bâtiment 210, Université de Paris-Sud, 91405 Orsay Cedex, France
}

(Received 24 September 1999)

\begin{abstract}
We model a disordered planar monolayer of paramagnetic spherical particles, or ferrofluid, as a twodimensional fluid of hard spheres with embedded three-dimensional magnetic point dipoles. This model, in which the orientational degrees of freedom are three dimensional while particle positions are confined to a plane, can be taken as a crude representation of a colloidal suspension of superparamagnetic particles confined in a water/air interface, a system that has recently been studied experimentally. In this paper, we propose an Ornstein-Zernike integral equation approach capable of describing the structure of this highly inhomogeneous fluid, including the effects of an external magnetic field. The method hinges on the use of specially tailored orthogonal polynomials whose weight function is precisely the one-particle distribution function that describes the surface- and field-induced anisotropy. The results obtained for various particle densities and external fields are compared with Monte Carlo simulations, illustrating the capability of the inhomogeneous Ornstein-Zernike equation and the proposed solution scheme to yield a detailed and accurate description of the spatial and orientational structure for this class of systems. For comparison, results from density-functional theory in the modified mean-field approximation are also presented; this latter approach turns out to yield at least qualitatively correct results.

PACS number(s): 61.20.Gy, 68.15.+e, 75.10.-b, 75.30.-m
\end{abstract}

\section{INTRODUCTION}

Magnetic films currently attract a great deal of interest because of their obvious potential for technological applications, especially given the progress in epitaxial growth techniques that has facilitated their manufacture. These systems consist of thin films (a few atomic layers) of paramagnetic particles (metal oxides in most cases) deposited on a solid surface (usually metallic). The competition between the different magnetic interactions and the constraint imposed on the particles to lie on a plane gives rise to a rich variety of phase transitions, from paramagnetic phases to in-plane or out-of-plane ferromagnetic and even antiferromagnetic phases [1]. The terms included in the Hamiltonian describing these systems stem from the magnetic dipole-dipole interaction, the exchange coupling, and the spin-orbit coupling that gives rise to a magnetic surface anisotropy [2]. In addition to this type of material, quasi-bidimensional systems can also be produced using confined colloids, with the added interest that both fluid and crystalline structures can be generated in such systems. In particular, Zahn, Méndez-Alcaraz, and Maret [3] have studied self-diffusion in two-dimensional colloids by means of an arrangement of superparamagnetic colloidal particles (doped with $\mathrm{Fe}_{2} \mathrm{O}_{3}$ ) confined to a water/air interface. An external magnetic field perpendicular to the surface can then be used to induce tunable repulsive interactions between the particles and thus separate out the effects of direct and hydrodynamic interactions on the diffusion process. Zahn, Lenke, and Maret [4] have further exploited this system to investigate the crystallization dynamics of two- dimensional colloids, an extremely active area of research dominated by the search for the Kosterlitz-Thoules-HalperinNelson-Young two-stage melting transition [5]. In these colloidal systems the leading interaction is the dipole-dipole potential, aside from the obvious excluded volume effects due to the particle cores. If the external field is sufficiently strong in relation to the interparticle interaction, the induced parallel alignment of the dipoles reduces the dipolar interaction to a simple, tunable radial repulsive interaction suited for the crystallization and diffusion studies presented in Refs. [3] and [4]. More generally, however, one must explicitly consider the full orientational nature of the dipole-dipole interaction.

We are not aware of any theoretical investigation of this type of disordered systems in which the particles carrying three-dimensional magnetic dipoles are constrained to a planar surface and interact with an external field. But in fact, besides simulation calculations-the preferred tool in the modeling of ordered phases like those described in Ref. [1] - and direct measurements via digital videomicroscopy $[3,4]$, these disordered systems are also tractable within the framework of the inhomogeneous Ornstein-Zernike (OZ) equation [6], an approach that has recently been successfully applied by the authors to describe order-disorder and gasliquid phase transitions in three-dimensional Heisenberg spin fluids $[7,8]$. In the present paper, we further pursue this approach and, as in Refs. [7] and [8], exploit the properties of specially constructed orthogonal polynomials whose weight function is precisely the one-particle distribution function of the anisotropic system. This general procedure has already 
been successfully applied to a variety of systems with internal degrees of freedom, such as polarizable particles $[9,10]$, polydisperse systems in three [11] and two [12] dimensions, and the Heisenberg spin systems mentioned above. In the present case, anisotropy could also be thought of as an internal degree of freedom, described by the polar angle of the dipoles with respect to the normal to the plane, and the system could then be viewed as a purely two-dimensional model with a fluctuating in-plane particle magnetic moment.

The ferrofluid monolayer will be modeled as a fluid of hard spheres in a plane with embedded three-dimensional point dipoles; additionally, an external field may be applied perpendicular to the plane. Obviously, this model is too simplistic to allow a direct comparison with the experiments, in particular since in the setup of Zahn et al. [3] the dipole moment is induced by the external field. This could be modeled by including polarizability $[9,10]$; although feasible, such an extension would considerably increase the computational burden and therefore at this stage is beyond the scope of our work. In our treatment, the single-particle angular distribution function, $f(\cos \theta)$, which describes the probability of finding a dipole at angle $\theta$ with the normal to the plane, will be the weight function of the special orthogonal polynomials used to expand the one-particle and two-particle correlation functions. This angular distribution function is connected to the intermolecular potentials and the pair distribution function $g(12)$ through a Born-Green (BG) equation [6]. The two-body function $g(12)$ in turn is studied in terms of an inhomogeneous OZ equation, to which we couple the optimized reference hypernetted-chain closure. The results obtained from this theoretical approach are compared with extensive data from Monte Carlo simulations. We find that the linked set of integral equations can furnish a remarkably accurate description of both the one-body and two-body structure of the two-dimensional fluid, as well as of its thermodynamics. On the other hand, density-functional theories (DFT) have been extensively applied to the study of first- and second-order transitions in three-dimensional dipolar fluids, particularly providing qualitative insight into the order-disorder (ferroelectric) transition in Stockmayer fluids [13]. Therefore, as an alternative, we also explore here the density-functional theory with a modified mean field (MMF) approximation for the free-energy functional [14]. This approximation, which is considerably less involved than the inhomogeneous $\mathrm{OZ}$ equation, provides only semiquantitative information on the thermodynamics and the single-particle structure. This stands in contrast to the relatively accurate results provided by the same method in the case of the ferromagnetic Heisenberg spin fluids [15].

The rest of the paper can be sketched as follows. In the next section, we describe in detail the solution algorithm, based on specially tailored orthogonal polynomials, for the coupled system formed by the inhomogeneous $\mathrm{OZ}$ equation for $g(12)$ and the $\mathrm{BG}$ equation for $f(\cos \theta)$. The essentials of the MMF approach applied to the dipolar monolayer problem are summarized in Sec. III, while details of the simulation procedure used to generate our standard (reference) data can be found in Sec. IV. Finally, Sec. V describes our most significant results and conclusions.

\section{INTEGRAL EQUATION FORMULATION}

The interaction energy for a configuration of $N$ sphere centers $\mathbf{r}_{j}$ with embedded three-dimensional magnetic moments $\boldsymbol{\mu}_{j}$ in the plane area $A$ is

$$
U=\sum_{i<j} u_{0}\left(r_{i j}\right)+\sum_{i<j} u_{d d}\left(\mathbf{r}_{i j}, \omega_{i}, \omega_{j}\right)-\sum_{j} \boldsymbol{\mu}_{j} \cdot \mathbf{B}_{0},
$$

where $u_{0}(r)$ is the hard sphere interaction for spheres of diameter $\sigma$,

$$
u_{d d}\left(\mathbf{r}_{12}, \omega_{1}, \omega_{2}\right)=-\frac{\mu^{2}}{r_{12}^{3}}\left[3\left(\hat{\mathbf{r}}_{12} \cdot \hat{\boldsymbol{\mu}}_{1}\right)\left(\hat{\mathbf{r}}_{12} \cdot \hat{\boldsymbol{\mu}}_{2}\right)-\hat{\boldsymbol{\mu}}_{1} \cdot \hat{\boldsymbol{\mu}}_{2}\right]
$$

the dipole-dipole potential, and $\mathbf{B}_{0}$ a uniform magnetic field perpendicular to the plane. Even in the absence of the magnetic field, the planar arrangement of the point dipoles produces anisotropy, so that the one-body density

$$
\rho^{(1)}(\mathbf{r}, \omega)=\left\langle\sum_{j=1}^{N} \delta\left(\mathbf{r}-\mathbf{r}_{j}\right) \delta\left(\omega-\omega_{j}\right)\right\rangle=\frac{\rho}{4 \pi} f(\omega)
$$

is not a constant. Here, $\rho=N / A$ is the number density and $f(\omega)$ the orientational distribution function of the interacting dipoles, which is to be found. Letting the magnetic field define the $z$ axis perpendicular to the plane, the one-body distribution for $\omega=(\theta, \varphi)$ depends only on the polar angle $\theta$, $f(\omega)=f(x)$, where $x=\cos \theta=\hat{\boldsymbol{\mu}} \cdot \hat{\mathbf{z}}$. [The distribution function in the noninteracting limit is $f_{0}(x)=\exp \left(\beta \mu B_{0} x\right) / C$, where $C=\sinh \left(\beta \mu B_{0}\right) / \beta \mu B_{0}$ is the normalization constant.] The two-body density

$$
\begin{aligned}
\rho^{(2)}\left(\mathbf{r}, \omega, \mathbf{r}^{\prime}, \omega^{\prime}\right)= & \left\langle\sum_{i \neq j} \delta\left(\mathbf{r}-\mathbf{r}_{i}\right) \delta\left(\omega-\omega_{i}\right)\right. \\
& \left.\times \delta\left(\mathbf{r}^{\prime}-\mathbf{r}_{j}\right) \delta\left(\omega^{\prime}-\omega_{j}\right)\right\rangle \\
& =\frac{\rho^{2}}{(4 \pi)^{2}} f(\omega) f\left(\omega^{\prime}\right) g\left(\mathbf{r}-\mathbf{r}^{\prime}, \omega, \omega^{\prime}\right)
\end{aligned}
$$

then defines the generalized pair distribution function $g(12)=g\left(\mathbf{r}_{12}, \omega_{1}, \omega_{2}\right)$. In the expressions above, a caret denotes unit vector while the angular brackets indicate a canonical ensemble average.

To determine $f(x)$ and $g(12)$ we proceed as in the earlier works [7-10]. The first BG equation,

$$
\begin{aligned}
\frac{d}{d x} \ln \left[\frac{f(\omega)}{f_{0}(\omega)}\right]= & -\frac{\rho}{4 \pi} \int d \mathbf{r} d \omega^{\prime} f\left(\omega^{\prime}\right) g\left(\mathbf{r}, \omega, \omega^{\prime}\right) \\
& \times \frac{d}{d x} \beta u_{d d}\left(\mathbf{r}, \omega, \omega^{\prime}\right),
\end{aligned}
$$

couples the one-body to the two-body distribution; here $\beta$ $=1 / k_{B} T$ is the inverse temperature. The pair function in turn is obtained from the inhomogeneous $\mathrm{OZ}$ equation 


$$
\begin{aligned}
h\left(\mathbf{r}_{12}, \omega_{1}, \omega_{2}\right)= & c\left(\mathbf{r}_{12}, \omega_{1}, \omega_{2}\right)+\frac{\rho}{4 \pi} \int d \mathbf{r}_{3} d \omega_{3} f\left(\omega_{3}\right) \\
& \times h\left(\mathbf{r}_{13}, \omega_{1}, \omega_{3}\right) c\left(\mathbf{r}_{32}, \omega_{3}, \omega_{2}\right),
\end{aligned}
$$

where $h=g-1$. The OZ equation introduces a second pair function, the generalized direct correlation function $c$, and so a second, or closure, relation between $c$ and $h$ is needed. This relation is [16]

$$
\begin{aligned}
c\left(\mathbf{r}, \omega_{1}, \omega_{2}\right)= & \exp \left[-\beta u_{0}(r)-\beta u_{d d}\left(\mathbf{r}, \omega_{1}, \omega_{2}\right)\right. \\
& \left.+\gamma\left(\mathbf{r}, \omega_{1}, \omega_{2}\right)+b\left(\mathbf{r}, \omega_{1}, \omega_{2}\right)\right] \\
& -1-\gamma\left(\mathbf{r}, \omega_{1}, \omega_{2}\right),
\end{aligned}
$$

where $\gamma=h-c$. [The indirect correlation function $\gamma$ will be used below to replace $h$ in Eq. (6) as the principal unknown.] It is here that the only approximation of the calculation must be introduced, for the so-called "bridge" function $b\left(\mathbf{r}, \omega_{1}, \omega_{2}\right)$ is not known in any usable form [16]. In this paper, we will use the reference hypernetted-chain (RHNC) closure $[17,18]$, optimized to achieve minimization of the free energy [19]. This choice consists in replacing the unknown function $b$ with the bridge function obtained from some calculable reference model, here taken to be the pure hard disk fluid at the same density,

$$
b\left(\mathbf{r}, \omega_{1}, \omega_{2}\right) \approx b_{\mathrm{HD}}\left(r ; \sigma_{0}\right),
$$

where $\sigma_{0}$ is the reference fluid disk diameter that is varied to attain a minimum in the free energy [19].

Equations (5)-(8) form a closed set; the problem now is to cast them into a computable form. This is achieved by expanding all angle-dependent functions in generalized spherical harmonics $[7,8]$

$$
\mathcal{Y}_{l m}(\omega)=\frac{1}{\sqrt{4 \pi}}(-1)^{m} e^{i m \varphi} \mathcal{P}_{l m}(\cos \theta)
$$

that are orthogonal with weight function $f(\omega)$,

$$
\int d \omega f(\omega) \mathcal{Y}_{l m}(\omega) \mathcal{Y}_{l^{\prime} m^{\prime}}^{*}(\omega)=\delta_{l l^{\prime}} \delta_{m m^{\prime}} .
$$

Since $f(\omega)$ here depends only on $\theta$, it is just the generalized Legendre functions $\mathcal{P}_{l m}(\cos \theta)$ that must be specially constructed. We refer to Ref. [8] for some formal details of their construction. Their actual calculation is based on a robust algorithm proposed by Press and Teukolsky [20].

Pair functions such as $\gamma$ are now expanded in this basis set as

$$
\begin{aligned}
\gamma\left(\mathbf{r}, \omega_{1}, \omega_{2}\right)= & 4 \pi \sum_{l_{1}, l_{2}, m_{1}, m_{2}} \gamma_{l_{1} l_{2}}^{m_{1} m_{2}}(r) \mathcal{Y}_{l_{1} m_{1}}\left(\omega_{1}\right) \\
& \times \mathcal{Y}_{l_{2} \bar{m}_{2}}\left(\omega_{2}\right) \exp \left[-i\left(m_{1}-m_{2}\right) \varphi_{r}\right],
\end{aligned}
$$

where $\varphi_{r}$ is the azimuthal angle of the planar vector $\mathbf{r}$ with the $x$ axis in the plane and $\bar{m}=-m$. In practice, it is convenient to align the $x$ axis along $\mathbf{r}$, so $\varphi_{r}=0$ and the expansion simplifies to

$$
\gamma\left(\mathbf{r}, \omega_{1}, \omega_{2}\right)=4 \pi \sum_{l_{1}, l_{2}, m_{1}, m_{2}} \gamma_{l_{1} l_{2}}^{m_{1} m_{2}}(r) \mathcal{Y}_{l_{1} m_{1}}\left(\omega_{1}\right) \mathcal{Y}_{l_{2} \bar{m}_{2}}\left(\omega_{2}\right)
$$

Similarly, Fourier transforms, which will be used to deconvolute the OZ Eq. (6), are also conveniently expanded with the $x$ axis along the planar vector $\mathbf{k}$. A two-dimensional Fourier transform

$$
\tilde{\gamma}(12)=\int d \mathbf{r} \gamma(12) e^{i \mathbf{k} \cdot \mathbf{r}}
$$

is then evaluated as follows. Choose the $x$ axis along $\mathbf{k}$. Then using Eq. (11) we have

$$
\begin{aligned}
\tilde{\gamma}(12)= & \int d \mathbf{r} \gamma(12) e^{i k r \cos \varphi_{r}} \\
= & 4 \pi \sum_{l_{1}, l_{2}, m_{1}, m_{2}} \int_{0}^{\infty} d r r \gamma_{l_{1} l_{2}}^{m_{1} m_{2}}(r) \int_{0}^{2 \pi} d \varphi_{r} e^{i k r \cos \varphi_{r}} \\
& \times e^{-i\left(m_{1}-m_{2}\right) \varphi_{r} \mathcal{Y}_{l_{1} m_{1}}\left(\omega_{1}\right) \mathcal{Y}_{l_{2} \bar{m}_{2}}\left(\omega_{2}\right)} \\
= & 4 \pi \sum_{l_{1}, l_{2}, m_{1}, m_{2}} \tilde{\gamma}_{l_{1} l_{2}}^{m_{1} m_{2}}(k) \mathcal{Y}_{l_{1} m_{1}}\left(\omega_{1}\right) \mathcal{Y}_{l_{2} \bar{m}_{2}}\left(\omega_{2}\right),
\end{aligned}
$$

where

$$
\tilde{\gamma}_{l_{1} l_{2}}^{m_{1} m_{2}}(k)=2 \pi i^{m_{1}-m_{2}} \int_{0}^{\infty} d r r \gamma_{l_{1} l_{2}}^{m_{1} m_{2}}(r) J_{\left|m_{1}-m_{2}\right|}(k r) .
$$

Here, $J_{m}(x)$ is the Bessel function of order $m$ generated by the integral over $\varphi_{r}$. Similarly, an inverse transform is

$$
\gamma_{l_{1} l_{2}}^{m_{1} m_{2}}(r)=\frac{1}{2 \pi i^{m_{1}-m_{2}}} \int_{0}^{\infty} d k k \tilde{\gamma}_{l_{1} l_{2}}^{m_{1} m_{2}}(k) J_{\left|m_{1}-m_{2}\right|}(k r) .
$$

It follows from the circular symmetry of the system that $\left|m_{1}-m_{2}\right|$ must be an even integer.

With these operations of Fourier transformation and expansion in generalized spherical harmonics, the OZ Eq. (6) is converted into a set of algebraic equations for the expansion coefficients,

$$
\tilde{\gamma}_{l_{1} l_{2}}^{m_{1} m_{2}}(k)=\rho \sum_{l_{3}, m_{3}}(-1)^{m_{3}}\left[\tilde{\gamma}_{l_{1} l_{3}}^{m_{1} m_{3}}(k)+\tilde{c}_{l_{1} l_{3}}^{m_{1} m_{3}}(k)\right] \widetilde{c}_{l_{3} l_{2}}^{m_{3} m_{2}}(k) .
$$

Coefficients with even or odd index pairs $\left(m_{1}, m_{2}\right)$ form disjoint sets, so Eq. (17) can be solved for the $\tilde{\gamma}_{l_{1} l_{2}}^{m_{1} m_{2}}(k)$ coefficients in the matrix forms

$$
\widetilde{\Gamma}(k)=\rho \widetilde{C}(k) \widetilde{C}(k)[I-\rho \widetilde{C}(k)]^{-1},
$$

for $m_{1}, m_{2}$ even, and

$$
\widetilde{\Gamma}(k)=-\rho \widetilde{C}(k) \widetilde{C}(k)[I+\rho \widetilde{C}(k)]^{-1},
$$

for $m_{1}, m_{2}$ odd. Here, $I$ is the unit matrix. The iteration scheme that is now used to solve for $f(x)$ and $g(12)$ follows 
that of earlier works [7-10], with the differences that arise from the reduced dimensionality having been spelled out above.

The thermodynamics of the system is then immediately obtainable by quadratures. For the internal energy $U_{d d}$, pressure $p$, and isothermal compressibility $K_{T}$, we get

$$
\begin{aligned}
\frac{\beta U_{d d}}{N}= & \frac{1}{2} \frac{\rho}{(4 \pi)^{2}} \int d \mathbf{r} d \omega_{1} d \omega_{2} f\left(\omega_{1}\right) f\left(\omega_{2}\right) \\
& \times g\left(\mathbf{r}, \omega_{1}, \omega_{2}\right) \beta u_{d d}\left(\mathbf{r}, \omega_{1}, \omega_{2}\right) \\
= & \frac{1}{2} \rho \int d \mathbf{r} \sum_{l_{1}, l_{2}, m_{1}, m_{2}} g_{l_{1} l_{2}}^{m_{1} m_{2}}(r) \beta u_{l_{1} l_{2}}^{m_{1} m_{2}}(r), \\
\frac{\beta p}{\rho}= & 1-\frac{1}{4} \frac{\rho}{(4 \pi)^{2}} \int d \mathbf{r} d \omega_{1} d \omega_{2} f\left(\omega_{1}\right) f\left(\omega_{2}\right) \\
& \times g\left(\mathbf{r}, \omega_{1}, \omega_{2}\right) r \frac{d}{d r} \beta u\left(\mathbf{r}, \omega_{1}, \omega_{2}\right) \\
= & 1+\frac{1}{2} \pi \rho \sigma^{2} g_{00}^{00}(\sigma)+\frac{3}{2} \frac{\beta U_{d d}}{N}, \\
& \quad \rho k_{B} T K_{T}=1+\rho \widetilde{h}_{00}^{00}(0) .
\end{aligned}
$$

The coefficients of the dipole-dipole potential (2) that appear in the energy calculation, Eq. (20), can be readily obtained. They are explicitly

$$
\begin{gathered}
\beta u_{00}^{00}(r)=\langle x\rangle^{2} \frac{\beta \mu^{2}}{r^{3}}, \\
\beta u_{01}^{00}(r)=\beta u_{10}^{00}(r)=\langle x\rangle \sigma_{x} \frac{\beta \mu^{2}}{r^{3}}, \\
\beta u_{11}^{00}(r)=\sigma_{x}^{2} \frac{\beta \mu^{2}}{r^{3}}, \\
\beta u_{11}^{11}(r)=\beta u_{11}^{-1-1}(r)=\frac{1}{4}\left(1-\left\langle x^{2}\right\rangle\right) \frac{\beta \mu^{2}}{r^{3}}, \\
\beta u_{11}^{1-1}(r)=\beta u_{11}^{-11}(r)=-\frac{3}{4}\left(1-\left\langle x^{2}\right\rangle\right) \frac{\beta \mu^{2}}{r^{3}},
\end{gathered}
$$

where $\sigma_{x}^{2}=\left\langle x^{2}\right\rangle-\langle x\rangle^{2}$ is the variance of $f(x)$. The virial pressure from these coefficients then obviously reduces to the dipole-dipole energy term seen in Eq. (21).

The potential coefficients are used in evaluating Eq. (5) expanded in the new orthogonal polynomials to yield [8]

$$
\frac{d}{d x} \ln \left[\frac{f(x)}{f_{0}(x)}\right]=\sum_{l_{1} l_{2} m} \xi_{l_{1} l_{2} m} \mathcal{P}_{l_{1} m}(x) \frac{d \mathcal{P}_{l_{2} m}(x)}{d x},
$$

with

$$
\xi_{l_{1} l_{2} m}=-\rho \int d \mathbf{r} \sum_{l_{3} m_{3}} g_{l_{1} l_{3}}^{m m_{3}}(r) u_{l_{3} l_{2}}^{m_{3} m}(r) .
$$

This expression can be recast in the form

$$
\ln f(x)=\ln f_{0}(x)+\sum_{l=0}^{\infty} a_{l} \mathcal{P}_{l 0}(x)
$$

where the $a_{l}$ for $l>0$ are determined by numerical (Gaussian) integration of Eq. (28) and $a_{0}$ by normalization.

Finally, with the magnetic field normal to the plane, the longitudinal (out-of-plane) and transverse (in-plane) magnetic susceptibilities are obtained, respectively, as [8]

$$
\begin{aligned}
\chi_{L} / \rho \beta \mu^{2}= & \sigma_{x}^{2}\left[1+\rho \widetilde{h}_{11}^{00}(0)\right]+\sigma_{x}\langle x\rangle\left[\rho \widetilde{h}_{10}^{00}(0)+\rho \widetilde{h}_{01}^{00}(0)\right] \\
& +\langle x\rangle^{2}\left[1+\rho \widetilde{h}_{00}^{00}(0)\right] \\
& \chi_{T} / \rho \beta \mu^{2}=\frac{1}{2}\left(1-\left\langle x^{2}\right\rangle\right)\left[1-\rho \widetilde{h}_{11}^{11}(0)\right]
\end{aligned}
$$

It was noted above that a hard disk fluid is used as the reference model for the optimized RHNC closure. Since the free energy minimization of the optimization procedure implicitly assumes thermodynamic consistency of the reference system, and no such solution is known for hard disks, we have sought at least to approximate that condition by using a pressure-consistent closure for the hard disk fluid [21],

$c(r)=h(r)-(1-\xi)\left[g(r) e^{\beta u_{\mathrm{HD}}(r)}-1\right]-\xi \ln \left[g(r) e^{\beta u_{\mathrm{HD}}(r)}\right]$.

This is a blend of the familiar Percus-Yevick $(\xi=0)$ and hypernetted-chain $(\xi=1)$ closures controlled by the parameter $\xi$ to achieve consistency of the virial and compressibility pressures. The density dependence of $\xi$ constrained in this way is found by calculation to be fitted by

$$
\xi=0.0920+0.1222 \rho^{*}+0.1642 \rho^{* 2}+0.1100 \rho^{* 3},
$$

with $\rho^{*} \equiv \rho \sigma^{2}$.

Reference [21] (see also Ref. [22]) lays out an accurate numerical algorithm for the Fourier-Hankel transform

$$
\tilde{\gamma}(k)=2 \pi \int_{0}^{\infty} d r r \gamma(r) J_{0}(k r)
$$

and its inverse. [The algorithm is based on the zeros of $J_{0}(x)$, an almost-periodic function, and so leads to unequal intervals in the numerical grids for $r$ and $k$.] Here, we need not only this transform but also those for $J_{m}(k r)$, with $m$ $>0$ an even integer. This has been handled in our calculation by a two-dimensional version of the "raising" and "lowering" operations previously used for spherical Bessel function transforms [23] that reduce all transforms to the $J_{0}(k r)$ variety. Specifically, to transform a coefficient $\gamma^{(m)}(r)$ $\equiv \gamma_{l_{1} l_{2}}^{m_{1} m_{2}}(r)$ for which $m \equiv\left|m_{1}-m_{2}\right|=2,4,6, \ldots$, we apply the lowering operation

$$
\gamma^{(m-2)}(r)=\gamma^{(m)}(r)-2(m-1) r^{m-2} \int_{r}^{\infty} d x \frac{\gamma^{(m)}(x)}{x^{m-1}}
$$

recursively until reaching $\gamma^{(0)}(r)$ and then transform this last function with the algorithm of Eq. (35). For an inverse trans- 
form, the function $\gamma^{(0)}(r)$ obtained from a $J_{0}(k r)$ inversion is subsequently raised to the final $\gamma_{l_{1} l_{2}}^{m_{1} m_{2}}(r)$ by recursive application of

$$
\gamma^{(m)}(r)=\gamma^{(m-2)}(r)-\frac{2(m-1)}{r^{m}} \int_{0}^{r} d x x^{m-1} \gamma^{(m-2)}(x)
$$

until reaching $m=\left|m_{1}-m_{2}\right|$. The integrals in Eqs. (36) and (37) are evaluated using a trapezoidal rule with unequal intervals. All calculations are carried out with 1000 points in the $r$ and $k$ grids.

Because the direct correlation function goes asymptotically as the potential,

$$
c_{l_{1} l_{2}}^{m_{1} m_{2}}(r) \sim-\beta u_{l_{1} l_{2}}^{m_{1} m_{2}}(r)
$$

for $r$ large, the calculation of the transforms of $c$ needed in Eq. (17) can be adversely affected by the long-range nature of the dipole potential, Eqs. (23)-(27), since the numerical versions of the transforms are necessarily truncated at a finite range. To avoid this problem, we subtract from the coefficients of $c$ a long-range function, appropriately weighted, that can be analytically transformed, namely,

$$
\begin{aligned}
\beta u_{\mathrm{LR}}^{(0)}(r) & =\frac{\beta \mu^{2}}{r^{3}}\left[1-e^{-\alpha r}\left(1+\alpha r+\frac{1}{2} \alpha^{2} r^{2}\right)\right] \\
& =\frac{1}{2} \beta \mu^{2} \alpha^{3} \int_{0}^{1} d x x^{2} e^{-\alpha r x}
\end{aligned}
$$

for those coefficients that transform with a $J_{0}(k r)$ kernel, Eqs. (23)-(26), and

$$
\begin{aligned}
\beta u_{\mathrm{LR}}^{(2)}(r) & =\frac{\beta \mu^{2}}{r^{3}}\left[1-e^{-\alpha r}\left(1+\alpha r+\frac{1}{2} \alpha^{2} r^{2}+\frac{1}{6} \alpha^{3} r^{3}\right)\right] \\
& =\frac{1}{6} \beta \mu^{2} \alpha^{4} r \int_{0}^{1} d x x^{3} e^{-\alpha r x},
\end{aligned}
$$

for those that transform with a $J_{2}(k r)$ kernel, Eq. (27). Here, $\alpha$ is a numerical parameter that we set at $\alpha=25 / R$, where $R=20 \sigma$ is the maximum range of $r$ in the calculation. The numerical transformation of the resulting short-range functions then presents no problems and the analytical transforms of $\beta u_{\mathrm{LR}}^{(0)}(r)$ and $\beta u_{\mathrm{LR}}^{(2)}(r)$ are added back in with the appropriate weights to complete the transformations.

\section{DENSITY-FUNCTIONAL THEORY IN MODIFIED MEAN FIELD APPROXIMATION}

As usual in DFT treatments, the free energy functional $F[\rho]$ is split into a reference part $F_{0}[\rho]$ corresponding to pure hard disks and a second contribution $F_{d d}[\rho]$ arising from the magnetic dipoles,

$$
F[\rho]=F_{0}[\rho]+F_{d d}[\rho] .
$$

The dipolar contribution in turn can be split into ideal and excess components,

$$
F_{d d}[\rho]=F_{d d}^{i d}+F_{d d}^{e x}[\rho],
$$

where the ideal part is simply

$$
\frac{\beta F_{d d}^{i d}}{N}=\frac{1}{4 \pi} \int d \omega f(\omega) \ln f(\omega)-\beta \mu B_{0}\langle x\rangle .
$$

As is found in Sec. V, we model the one-body function as

$$
f(\omega)=f(x)=\frac{1}{C} e^{-a x^{2}+b x},
$$

with the normalization constant

$$
C=\frac{1}{4} \sqrt{\frac{\pi}{a}} e^{b^{2} / 4 a}\left[\operatorname{erf}\left(\frac{2 a-b}{2 \sqrt{a}}\right)+\operatorname{erf}\left(\frac{2 a+b}{2 \sqrt{a}}\right)\right] .
$$

Here $\operatorname{erf}(x)$ is the standard error function and we have put $a \equiv-\beta \mu^{2} K, b \equiv \beta \mu B$, where $K$ and $B$ are effective values of the magnetic surface anisotropy and external magnetic field, respectively. Now, from Eq. (43), it is straightforward to show that

$$
\beta F_{d d}^{i d} / N=-a\left\langle x^{2}\right\rangle+\left(b-b_{0}\right)\langle x\rangle-\ln C .
$$

Analytic expressions for the moments $\left\langle x^{n}\right\rangle$ can be readily derived. Equation (46) is somewhat ill conditioned for calculations at small values of $a$, since the expression for $C$ contains both vanishing and diverging terms that have to be handled analytically. It is then advisable to perform a series expansion around $a=0$, which is rapidly convergent.

The excess term has now to be approximated. We follow Teixeira and Telo da Gama [14] in applying the modified mean field approximation, which leads to the excess free energy expression

$$
\begin{aligned}
\frac{\beta F_{d d}^{e x}}{N}= & \frac{\rho}{(4 \pi)^{2}} \int_{r>\sigma} d \mathbf{r} d \omega_{1} d \omega_{2} f\left(\omega_{1}\right) f\left(\omega_{2}\right) \\
& \times\left\{1-\exp \left[-\beta u_{d d}\left(\mathbf{r}, \omega_{1}, \omega_{2}\right)\right]\right\} .
\end{aligned}
$$

This integral is evaluated numerically employing Gaussian quadrature on the angular variables and a simple trapezoidal rule on $r$ [8], just as in the numerical algorithm for solving the integral equation in Sec. II.

For given $\rho, \mu$, and $B_{0}$, one has now to minimize the free energy defined by Eqs. (41)-(47) with respect to the parameters $a$ and $b$. The search for the minimum of a function defined in terms of a numerical multidimensional quadrature is an ill-conditioned problem and so Newton-Raphson or conjugate gradient algorithms are not well suited. We have found that a direct search Complex algorithm [24] provides a sufficiently robust method.

Finally, the excess internal energy in the MMF approximation must be evaluated by numerical integration of

$$
\begin{aligned}
\frac{\beta U_{d d}}{N}= & \frac{1}{2} \frac{\rho}{(4 \pi)^{2}} \int_{r>\sigma} d \mathbf{r} d \omega_{1} d \omega_{2} f\left(\omega_{1}\right) f\left(\omega_{2}\right) \\
& \times \exp \left[-\beta u_{d d}\left(\mathbf{r}, \omega_{1}, \omega_{2}\right)\right] \beta u_{d d}\left(\mathbf{r}, \omega_{1}, \omega_{2}\right) .
\end{aligned}
$$




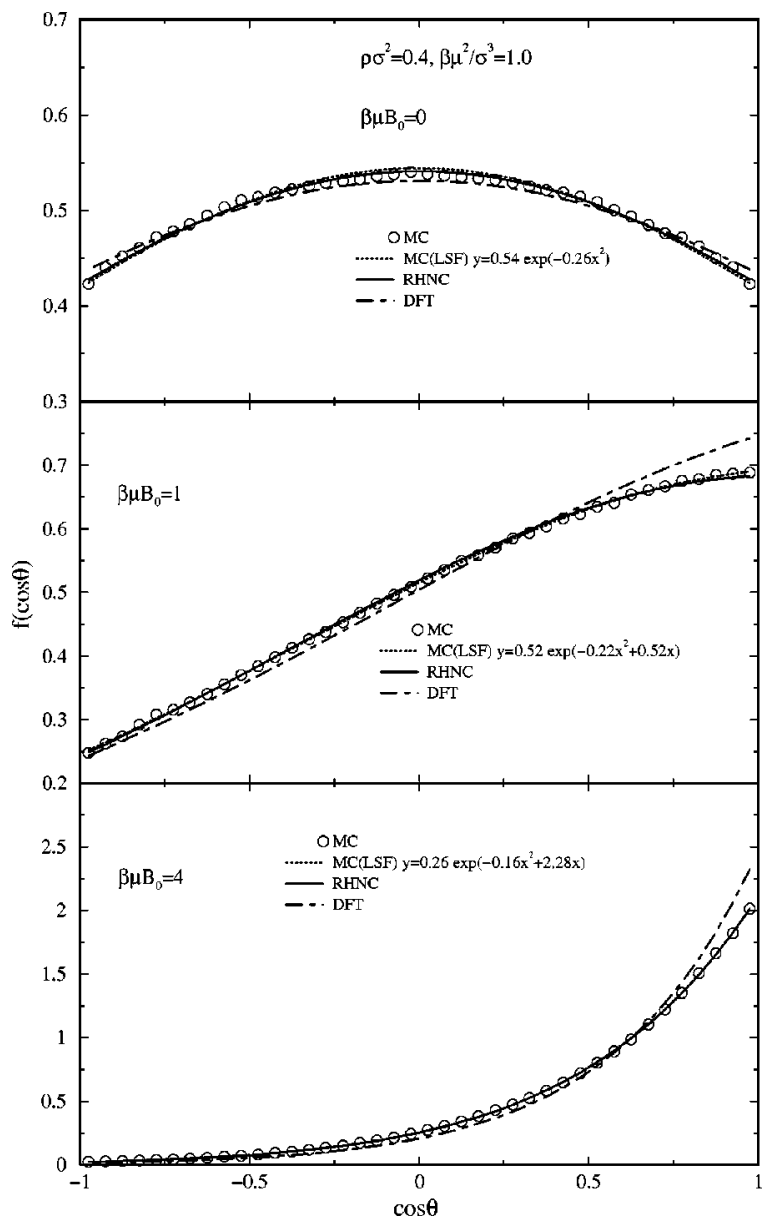

FIG. 1. Angular distribution function $f(\cos \theta)$ in a ferrofluid monolayer of dipolar hard spheres at $\rho \sigma^{2}=0.4$ and various external fields. Open circles denote MC data, solid line RHNC results, and dash-dotted line results from DFT-MMF. The dotted line tracking the MC points is a least squares fit (LSF) whose coefficients are shown in the figure.

\section{MONTE CARLO (MC) SIMULATION}

The MC simulations were performed in the canonical $(N V T)$ ensemble using 576 particles in a square box with periodic boundary conditions. The long range of the dipole interaction was accounted for by an Ewald sum, which for a three-dimensional dipole interaction with particle centers restricted to a two-dimensional periodic lattice is absolutely convergent. We have found that the results exhibit no dependence on the boundary conditions, since for threedimensional dipoles the potential decays as $1 / r^{3}$, in contrast with the pure two-dimensional system with two-dimensional electrostatics in which the interactions decay as $1 / r^{2}$.

In deriving the expression for the Ewald sum, it is convenient to divide the dipole moment into an in-plane component $\boldsymbol{\mu}_{i}^{\|}$and an out-of-plane component $\mu_{i}^{\perp}$. The total energy can then be written as the sum of two contributions [25],

$$
U_{d d}=U_{\|}+U_{\perp},
$$

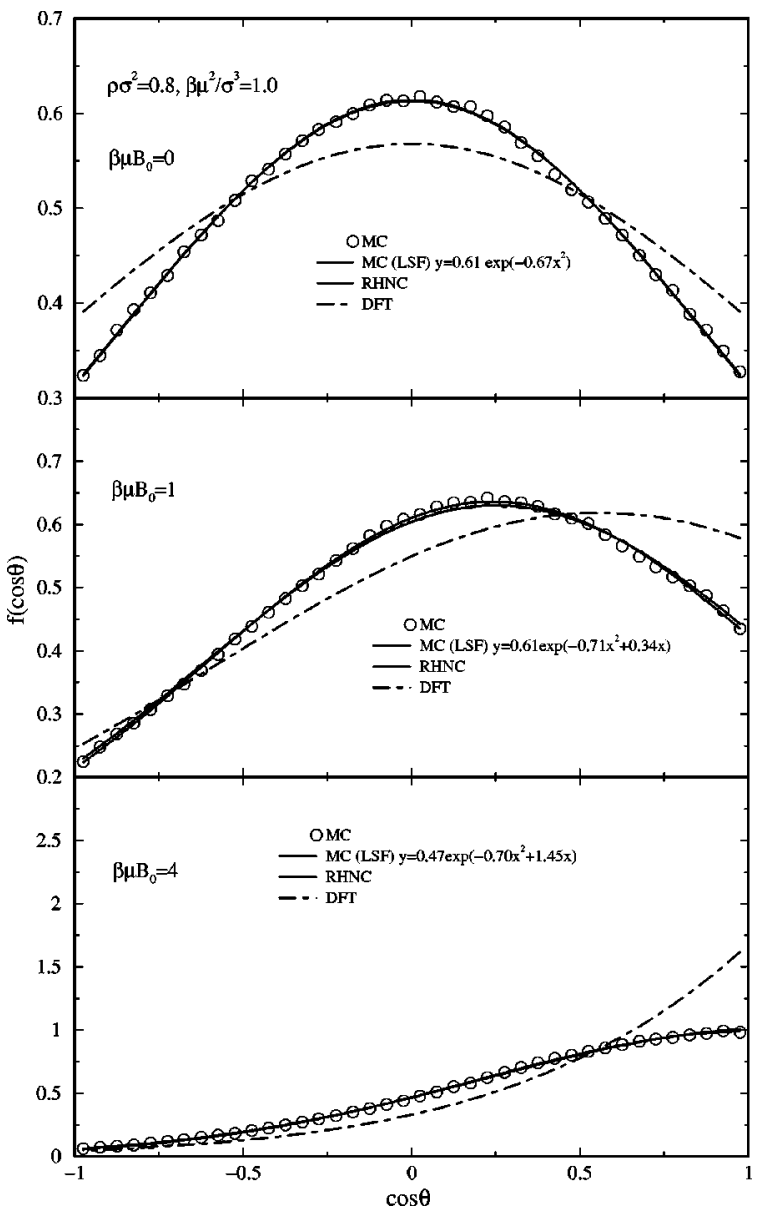

FIG. 2. Angular distribution function $f(\cos \theta)$ in a ferrofluid monolayer of dipolar hard spheres at $\rho \sigma^{2}=0.8$ and various external fields. Labels as in Fig. 1.

$$
\begin{aligned}
U_{\|}= & -\frac{1}{2} \sum_{i=1}^{N} \sum_{j=1}^{N} \sum_{\boldsymbol{n}}{ }^{\prime}\left[B\left(\left|\boldsymbol{r}_{i j}+\boldsymbol{n}\right|\right) \boldsymbol{\mu}_{i}^{\|} \cdot \boldsymbol{\mu}_{j}^{\|}+C\left(\left|\boldsymbol{r}_{i j}+\boldsymbol{n}\right|\right)\right. \\
& \left.\times\left(\boldsymbol{\mu}_{i}^{\|} \cdot \boldsymbol{r}_{i j}\right)\left(\boldsymbol{\mu}_{j}^{\|} \cdot \boldsymbol{r}_{i j}\right)\right]+\frac{\pi}{A} \sum_{\boldsymbol{G} \neq 0} \frac{\operatorname{erfc}(G / 2 \alpha)}{G} F_{\|}(\boldsymbol{G}) F_{\|}^{*}(\boldsymbol{G}) \\
& -\frac{2 \alpha^{3}}{3 \sqrt{\pi}} \sum_{i=1}^{N}\left(\mu_{i}^{\|}\right)^{2}
\end{aligned}
$$

and

$$
\begin{aligned}
U_{\perp}= & -\frac{1}{2} \sum_{i=1}^{N} \sum_{j=1}^{N} \sum_{\boldsymbol{n}}{ }^{\prime} B\left(\left|\boldsymbol{r}_{i j}+\boldsymbol{n}\right|\right) \mu_{i}^{\perp} \mu_{j}^{\perp} \\
& +\frac{\pi}{A} \sum_{\boldsymbol{G} \neq 0}\left[\frac{2 \alpha}{\sqrt{\pi}} \exp \left(-\frac{G^{2}}{4 \alpha^{2}}\right)-G \operatorname{erfc}\left(\frac{G}{2 \alpha}\right)\right] \\
& \times F_{\perp}(\boldsymbol{G}) F_{\perp}^{*}(\boldsymbol{G}) \\
& +\frac{2 \sqrt{\pi} \alpha}{A} \sum_{i=1}^{N} \sum_{j=1}^{N} \mu_{i}^{\perp} \mu_{j}^{\perp}-\frac{2 \alpha^{3}}{3 \sqrt{\pi}} \sum_{i=1}^{N}\left(\mu_{i}^{\perp}\right)^{2} .
\end{aligned}
$$

where 


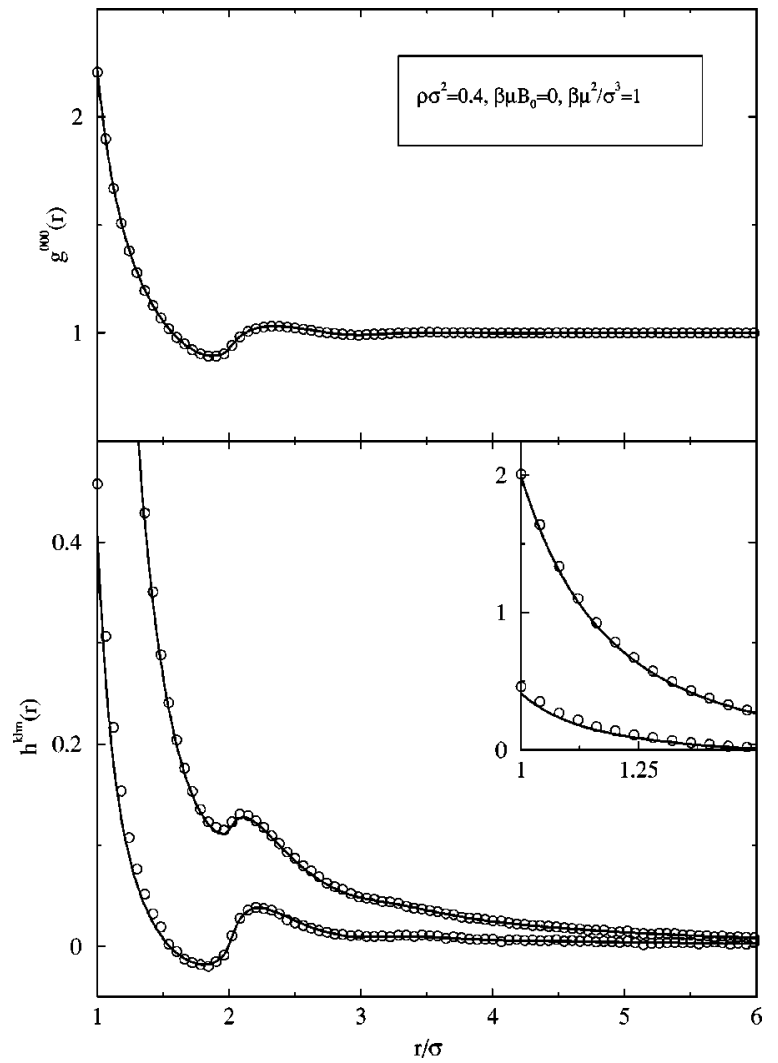

FIG. 3. Radial projection $g^{000}(r)$ and angular projections $h^{k l m}(r)=h^{110}(r)$ and $h^{112}(r)$ of the pair distribution function $g(12)$ in a ferrofluid monolayer of dipolar hard spheres at $\rho \sigma^{2}=0.4$ and $\beta \mu B_{0}=0$. Here and in Figs. $4-8$, the angular projection curve with the largest contact value corresponds to $h^{112}(r)$ for $\beta \mu B_{0}=0$ and $h^{110}(r)$ for $\beta \mu B_{0}=4$. Open circles denote MC data and solid lines represent RHNC results.

$$
\begin{gathered}
B(r)=-\frac{\operatorname{erfc}(\alpha r)}{r^{3}}-\frac{2 \alpha}{\sqrt{\pi}} \frac{\exp \left(-\alpha^{2} r^{2}\right)}{r^{2}}, \\
C(r)=3 \frac{\operatorname{erfc}(\alpha r)}{r^{5}}+\frac{2 \alpha}{\sqrt{\pi}}\left(\frac{3}{r^{2}}+2 \alpha^{2}\right) \frac{\exp \left(-\alpha^{2} r^{2}\right)}{r^{2}},
\end{gathered}
$$

while

$$
\begin{gathered}
F_{\|}(\boldsymbol{G})=\sum_{i=1}^{N}\left(\boldsymbol{G} \cdot \boldsymbol{\mu}_{i}^{\|}\right) \exp \left[i \boldsymbol{G} \cdot \boldsymbol{r}_{i}\right] \\
F_{\perp}(\boldsymbol{G})=\sum_{i=1}^{N} \mu_{i}^{\perp} \exp \left[i \boldsymbol{G} \cdot \boldsymbol{r}_{i}\right] .
\end{gathered}
$$

In Eqs. (50) and (51), $A=L^{2}$ is the area of the system and $L$ the box edge length. The prime in the sum over $\boldsymbol{n}$ $=\left(n_{x}, n_{y}\right)$, with $n_{x}, n_{y}$ integers, means that $i \neq j$ for $\boldsymbol{n}=\mathbf{0}$. With the value $\alpha=5.7 / L$ that was adopted in our calculations, only the terms with $\boldsymbol{n}=\mathbf{0}$ need be retained in Eqs. (50) and (51). The sum in reciprocal space extends over all lattice vectors $\boldsymbol{G}=2 \pi \boldsymbol{n} / L$ such that $|\boldsymbol{n}|^{2} \leqslant n_{\max }^{2}=64$. Because of the rather large system size the reciprocal space contribution to the energy is generally quite small.

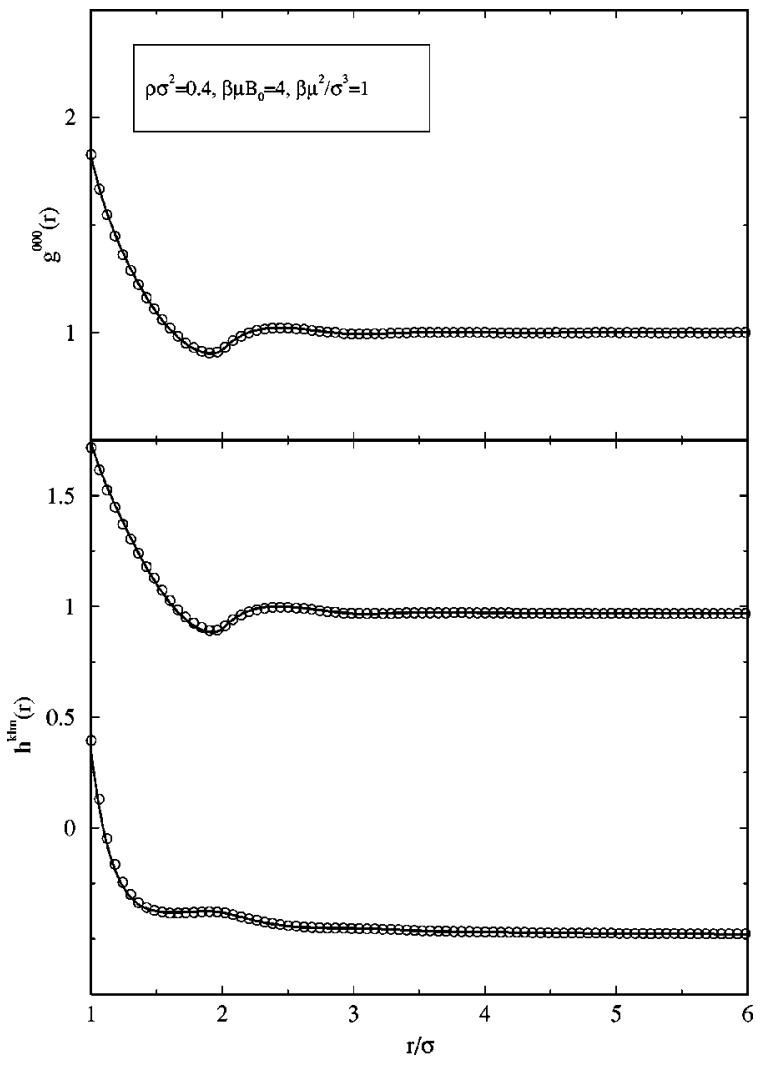

FIG. 4. Same as Fig. 3 for $\rho \sigma^{2}=0.4$ and $\beta \mu B_{0}=4$.

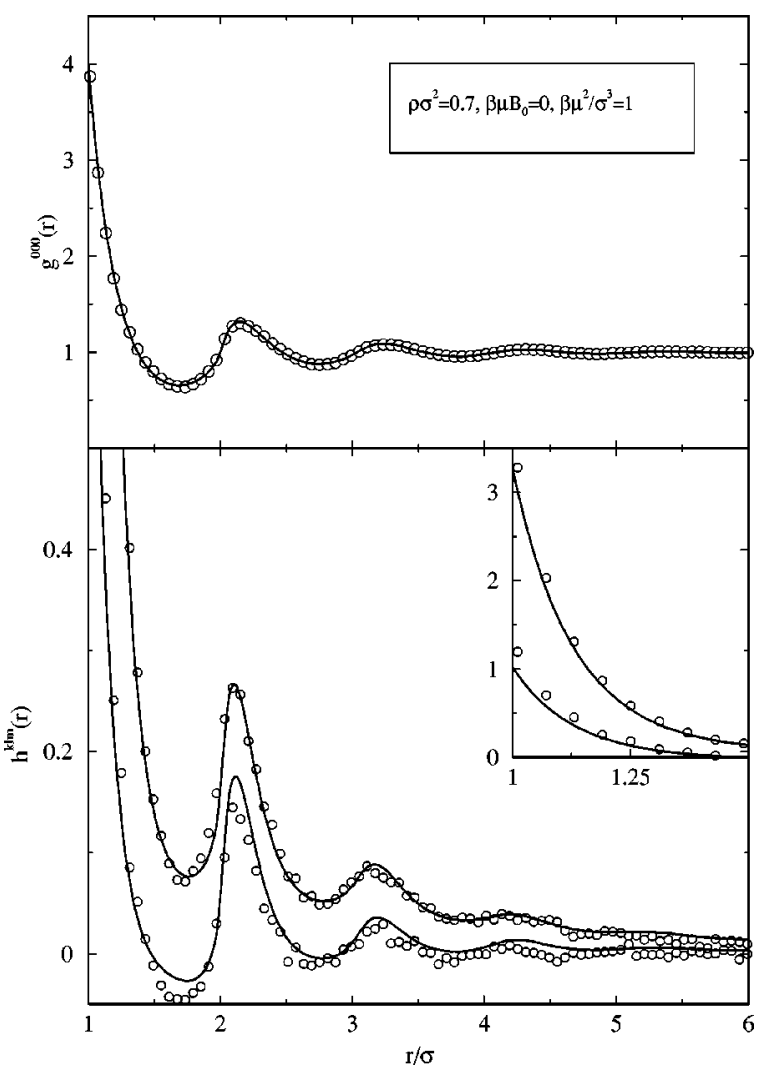

FIG. 5. Same as Fig. 3 for $\rho \sigma^{2}=0.7$ and $\beta \mu B_{0}=0$. 


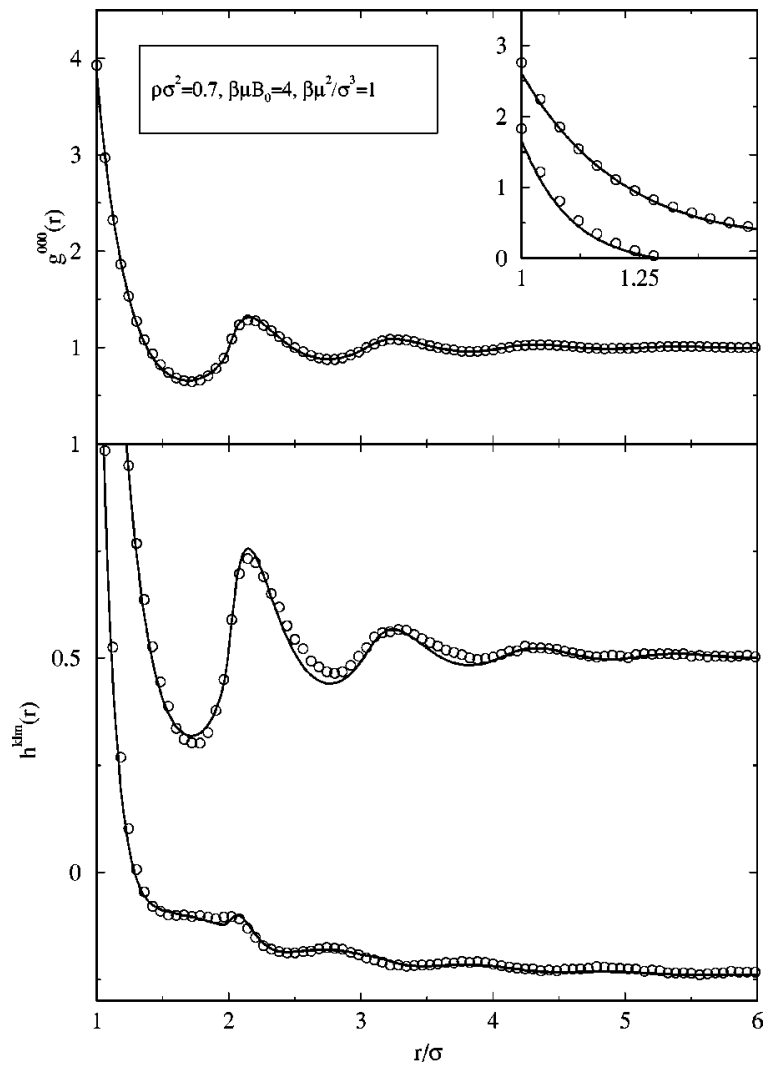

FIG. 6. Same as Fig. 3 for $\rho \sigma^{2}=0.7$ and $\beta \mu B_{0}=4$.

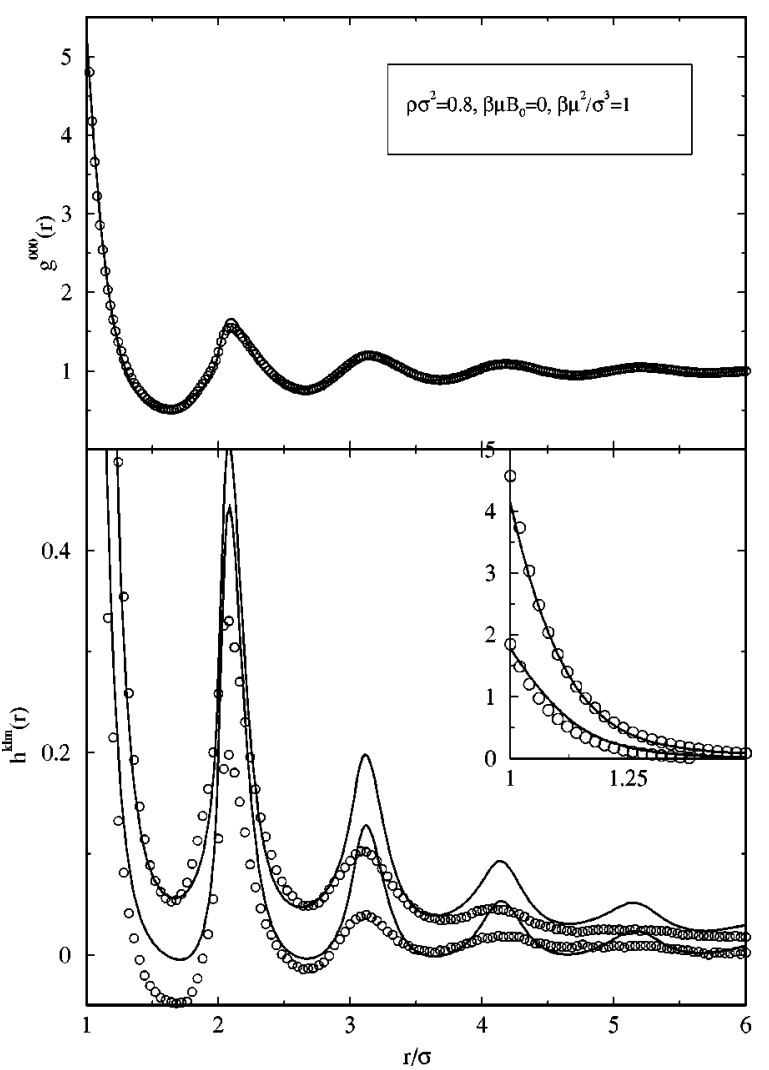

FIG. 7. Same as Fig. 3 for $\rho \sigma^{2}=0.8$ and $\beta \mu B_{0}=0$.

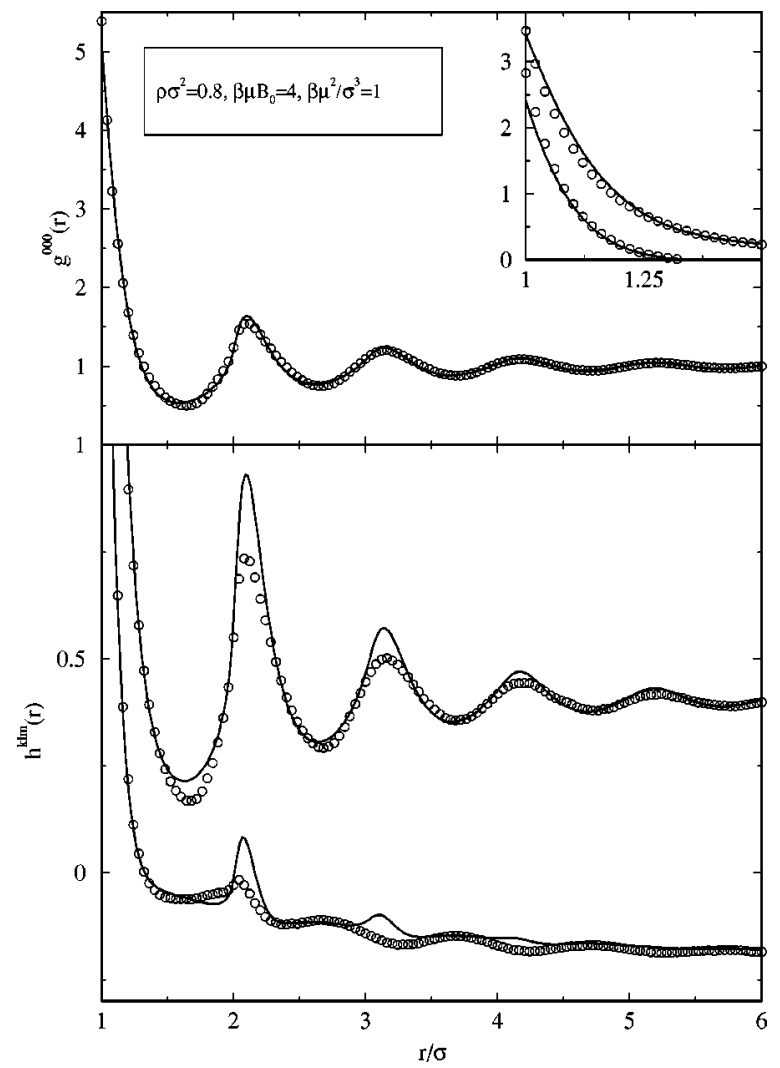

FIG. 8. Same as Fig. 3 for $\rho \sigma^{2}=0.8$ and $\beta \mu B_{0}=4$.

In the calculations presented in this work, simulation runs consisted of 50000 to 100000 cycles, each cycle implying a translation, rotation, and occasional inversion of the dipoles moment, carried out on every particle in the sample. The real space contribution in Eqs. (47) and (48) was truncated at half the box length. In order to assess the system size dependence of the results, some calculations were performed with 1600particle samples; they showed no significant deviations from the smaller sample results.

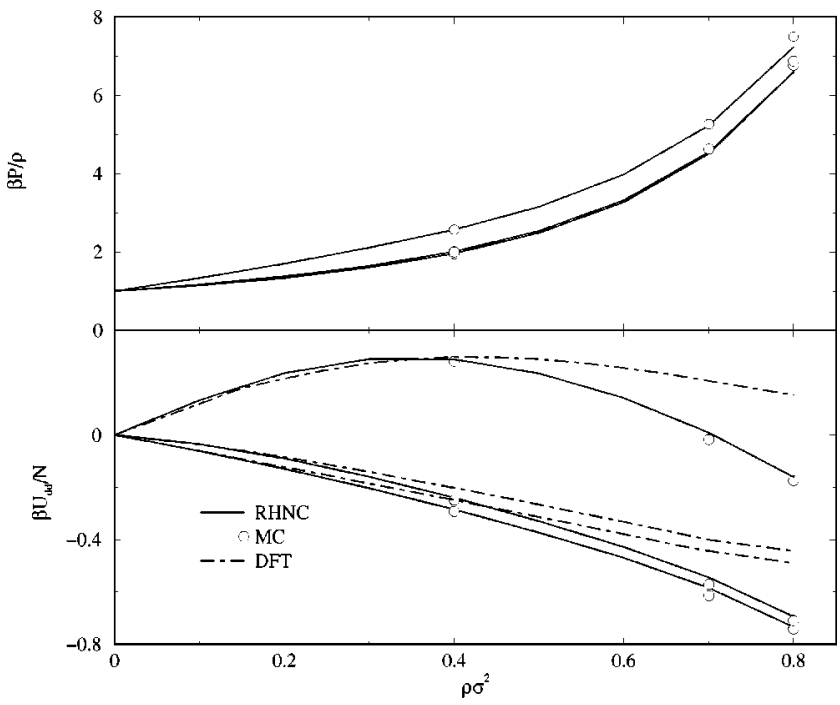

FIG. 9. Compressibility factor $\beta p / \rho$ and excess internal energy $\beta U_{d d} / N$ for a ferrofluid monolayer of dipolar hard spheres. Open circles denote MC data, solid line RHNC results, and dash-dotted line results from DFT-MMF. The curves, from bottom to top, correspond to $\beta \mu B_{0}=0,1$, and 4 . 
TABLE I. Magnetic and thermodynamic properties of a planar ferrofluid monolayer of dipolar hard spheres of diameter $\sigma$ with embedded point dipoles $\mu$ in an external magnetic field $B_{0}$ normal to the plane, calculated in optimized RHNC approximation for $\beta \mu^{2} / \sigma^{3}=1 ; \sigma_{0}$ is the optimized disk diameter of the reference hard disk system.

\begin{tabular}{cccccccccc}
\hline \hline$\rho \sigma^{2}$ & $\beta \mu B_{0}$ & $\sigma_{0} / \sigma$ & $\beta \mu B$ & $\beta \mu^{2} K$ & $\beta U_{d d} / N$ & $\beta p / \rho$ & $\beta \partial p / \partial \rho$ & $\chi_{L} / \rho \beta \mu^{2}$ & $\chi_{T} / \rho \beta \mu^{2}$ \\
\hline 0.1 & 0 & 0.9587 & 0 & -0.048 & -0.061 & 1.147 & 1.314 & 0.270 & 0.376 \\
0.2 & 0 & 0.9658 & 0 & -0.105 & -0.129 & 1.340 & 1.783 & 0.223 & 0.425 \\
0.3 & 0 & 0.9731 & 0 & -0.172 & -0.204 & 1.600 & 2.506 & 0.188 & 0.480 \\
0.4 & 0 & 0.9799 & 0 & -0.248 & -0.286 & 1.962 & 3.670 & 0.161 & 0.543 \\
0.5 & 0 & 0.9853 & 0 & -0.332 & -0.375 & 2.486 & 5.621 & 0.139 & 0.616 \\
0.6 & 0 & 0.9900 & 0 & -0.425 & -0.474 & 3.274 & 9.054 & 0.121 & 0.711 \\
0.7 & 0 & 0.9934 & 0 & -0.530 & -0.585 & 4.508 & 15.42 & 0.106 & 0.873 \\
0.8 & 0 & 0.9950 & 0 & -0.673 & -0.731 & 6.523 & 27.83 & 0.094 & 1.481 \\
0.1 & 1 & 0.9718 & 0.826 & -0.043 & -0.034 & 1.172 & 1.358 & 0.277 & 0.358 \\
0.2 & 1 & 0.9729 & 0.695 & -0.099 & -0.089 & 1.379 & 1.840 & 0.222 & 0.409 \\
0.3 & 1 & 0.9770 & 0.596 & -0.167 & -0.159 & 1.645 & 2.564 & 0.186 & 0.466 \\
0.4 & 1 & 0.9820 & 0.518 & -0.244 & -0.240 & 2.010 & 3.725 & 0.159 & 0.531 \\
0.5 & 1 & 0.9859 & 0.457 & -0.329 & -0.331 & 2.537 & 5.670 & 0.138 & 0.605 \\
0.6 & 1 & 0.9902 & 0.407 & -0.423 & -0.432 & 3.322 & 9.091 & 0.120 & 0.701 \\
0.7 & 1 & 0.9936 & 0.367 & -0.528 & -0.545 & 4.554 & 15.46 & 0.106 & 0.861 \\
0.8 & 1 & 0.9950 & 0.336 & -0.672 & -0.694 & 6.588 & 27.90 & 0.094 & 1.458 \\
0.1 & 4 & 1.0716 & 3.516 & 0.001 & 0.133 & 1.335 & 1.687 & 0.348 & 0.218 \\
0.2 & 4 & 1.0393 & 3.053 & -0.030 & 0.237 & 1.705 & 2.469 & 0.225 & 0.259 \\
0.3 & 4 & 1.0180 & 2.640 & -0.091 & 0.291 & 2.110 & 3.380 & 0.164 & 0.312 \\
0.4 & 4 & 1.0054 & 2.290 & -0.175 & 0.288 & 2.574 & 4.603 & 0.133 & 0.378 \\
0.5 & 4 & 0.9994 & 2.001 & -0.275 & 0.233 & 3.158 & 6.531 & 0.116 & 0.457 \\
0.6 & 4 & 0.9969 & 1.766 & -0.384 & 0.138 & 3.975 & 9.902 & 0.105 & 0.555 \\
0.7 & 4 & 0.9963 & 1.575 & -0.504 & 0.009 & 5.228 & 16.21 & 0.097 & 0.702 \\
0.8 & 4 & 0.9970 & 1.424 & -0.651 & -0.161 & 7.230 & 28.77 & 0.089 & 1.121 \\
\hline \hline
\end{tabular}

\section{RESULTS AND CONCLUSIONS}

One of the most significant structural quantities in an inhomogeneous system is obviously the one-particle distribution function, in this case the angular distribution function $f(x)$. Using Eqs. (28)-(30) we find by calculation that

$f(x)=\frac{1}{C} \exp \left(\beta \mu^{2} K x^{2}+\beta \mu B x\right)$, captures the orientational order of the system. In Eq. (56), $K$ and $B$ are effective values of the magnetic surface anisotropy and external magnetic field, respectively, while the normalization constant $C$ is given in Eq. (45). A comparison between our Monte Carlo data for $f(x)$ and results from the RHNC integral equation and the MMF approximation is displayed in Figs. 1 and 2 for various densities and external fields. Given that the magnitude of common magnetic dipoles is appreciably smaller than that of their electric coun-

TABLE II. Comparison of magnetic and thermodynamic properties of a planar ferrofluid monolayer of dipolar hard spheres in an external magnetic field $B_{0}$ normal to the plane, calculated in optimized RHNC approximation, Monte Carlo (MC) simulation, and density functional theory (DFT), for $\beta \mu^{2} / \sigma^{3}=1$.

\begin{tabular}{llllllllllllll}
\hline \hline$\rho \sigma^{2}$ & \multirow{2}{*}{$\beta \mu B_{0}$} & \multicolumn{3}{c}{$\beta \mu B$} & \multicolumn{4}{c}{$\beta \mu^{2} K$} & \multicolumn{4}{c}{$\beta U_{d d} / N$} & \multicolumn{3}{c}{$\beta p / \rho$} \\
& & RHNC & MC & DFT & RHNC & MC & DFT & RHNC & MC & DFT & RHNC & MC \\
\hline 0.4 & 0 & 0 & 0 & 0 & -0.248 & -0.26 & -0.202 & -0.286 & -0.292 & -0.248 & 1.962 & 1.95 \\
0.7 & 0 & 0 & 0 & 0 & -0.530 & -0.60 & -0.400 & -0.585 & -0.614 & -0.443 & 4.508 & 4.60 \\
0.8 & 0 & 0 & 0 & 0 & -0.673 & -0.67 & -0.393 & -0.731 & -0.742 & -0.489 & 6.523 & 6.76 \\
0.4 & 1 & 0.518 & 0.52 & 0.572 & -0.244 & -0.22 & -0.180 & -0.240 & -0.248 & -0.206 & 2.010 & 2.00 \\
0.7 & 1 & 0.367 & 0.38 & 0.356 & -0.528 & -0.53 & -0.435 & -0.545 & -0.571 & -0.401 & 4.554 & 4.63 \\
0.8 & 1 & 0.336 & 0.34 & 0.383 & -0.672 & -0.71 & -0.426 & -0.694 & -0.709 & -0.443 & 6.588 & 6.87 \\
0.4 & 4 & 2.290 & 2.28 & 2.578 & -0.175 & -0.16 & -0.100 & 0.288 & 0.282 & 0.275 & 2.574 & 2.57 \\
0.7 & 4 & 1.575 & 1.58 & 1.887 & -0.504 & -0.51 & -0.148 & 0.009 & -0.017 & 0.190 & 5.228 & 5.26 \\
0.8 & 4 & 1.424 & 1.45 & 1.802 & -0.651 & -0.70 & -0.177 & -0.161 & -0.175 & 0.153 & 7.230 & 7.50 \\
\hline \hline
\end{tabular}




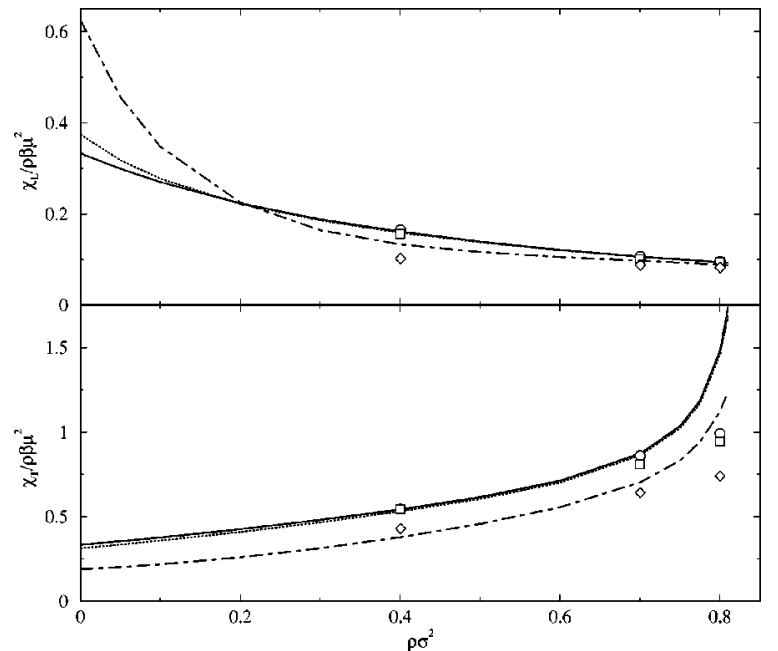

FIG. 10. Longitudinal and transverse magnetic susceptibilities of a ferrofluid monolayer in the RHNC approximation (lines) and from MC simulation (symbols). Solid lines (circles) correspond to $\beta \mu B_{0}=0$, dotted lines (squares) to $\beta \mu B_{0}=1$, and dash-dotted lines (diamonds) to $\beta \mu B_{0}=4$. The error on the $\mathrm{MC}$ results for the susceptibilities is about $5 \%$.

terparts, we have only considered a relatively moderate reduced dipole, $\mu^{*}=\left(\beta \mu^{2} / \sigma^{3}\right)^{1 / 2}=1$. In all cases, the agreement seen in the figures between the integral equation and the simulation results is excellent. The MMF approach, despite its crude assumptions, can be considered semiquantitative. The most noticeable feature observed in these figures is the breaking of the symmetry of the distribution with respect to the plane $(x=\cos \theta=0)$ due to the external field, with the response of the dipoles to the field being weaker at higher densities. This effect of density is readily understandable, since the dipole-dipole interaction favoring head-to-tail alignments increases the tendency of the dipoles to remain in-plane. The same effect can also be seen in the zero-field truncated Gaussian distributions of Figs. 1 and 2, which become progressively more sharply peaked about $x$ $=0$ as the density increases, approaching a fully coplanar distribution. A similar effect can be induced by an increase of the dipole moment.

We now focus on the pair structure of the system. In dipolar fluids, in addition to the center-to-center pair distribution function $g^{000}(r) \equiv g_{00}^{00}(r)$, the most significant projections of $g(12)$ are those onto the standard rotational invari- ants $\boldsymbol{\phi}^{110}=\hat{\boldsymbol{\mu}}_{1} \cdot \hat{\boldsymbol{\mu}}_{2} \quad$ and $\quad \boldsymbol{\phi}^{112}=3\left(\hat{\boldsymbol{\mu}}_{1} \cdot \hat{\mathbf{r}}\right)\left(\hat{\boldsymbol{\mu}}_{2} \cdot \hat{\mathbf{r}}\right)-\hat{\boldsymbol{\mu}}_{1} \cdot \hat{\boldsymbol{\mu}}_{2}$, namely, $h^{110}(r)$ and $h^{112}(r)$, quantities that are connected respectively to the relative orientation of a pair of dipoles at a given separation and to the contribution of a given orientation to the dipolar excess energy. In terms of the calculated coefficients $g_{l_{1} l_{2}}^{m_{1} m_{2}}(r)$ of this paper, these projections are given by

$$
\begin{aligned}
h^{110}(r) \equiv & 3\left\langle g(12) \boldsymbol{\phi}^{110}(12)\right\rangle_{\omega_{1} \omega_{2}} \\
= & 3\left[\langle x\rangle^{2} g_{00}^{00}(r)+2\langle x\rangle \sigma_{x} g_{01}^{00}(r)\right. \\
& \left.+\sigma_{x}^{2} g_{11}^{00}-\left(1-\left\langle x^{2}\right\rangle\right) g_{11}^{11}(r)\right]
\end{aligned}
$$

and

$$
\begin{aligned}
h^{112}(r) \equiv & \frac{3}{2}\left\langle g(12) \boldsymbol{\phi}^{112}(12)\right\rangle_{\omega_{1} \omega_{2}} \\
= & -\frac{3}{2}\left[\langle x\rangle^{2} g_{00}^{00}(r)+2\langle x\rangle \sigma_{x} g_{01}^{00}(r)+\sigma_{x}^{2} g_{11}^{00}(r)\right. \\
& \left.+\frac{1}{2}\left(1-\left\langle x^{2}\right\rangle\right) g_{11}^{11}(r)-\frac{3}{2}\left(1-\left\langle x^{2}\right\rangle\right) g_{11}^{1-1}(r)\right] .
\end{aligned}
$$

Note that these standard projections do not vanish at large separations, in contrast to the coefficients $h_{l_{1} l_{2}}^{m_{1} m_{2}}(r)$ obtained in the special orthogonal polynomial expansion. In fact, it follows from the equations above that they have the limiting behavior

$$
\begin{gathered}
\lim _{r \rightarrow \infty} h^{110}(r)=3\langle x\rangle^{2}, \\
\lim _{r \rightarrow \infty} h^{112}(r)=-\frac{3}{2}\langle x\rangle^{2} .
\end{gathered}
$$

The dipole-dipole energy, Eq. (20), can be obtained directly from $h^{112}(r)$ in the form

$$
\frac{\beta U_{d d}}{N}=-\frac{2}{3} \pi \rho \int_{0}^{\infty} d r h^{112}(r) \frac{\beta \mu^{2}}{r^{2}} .
$$

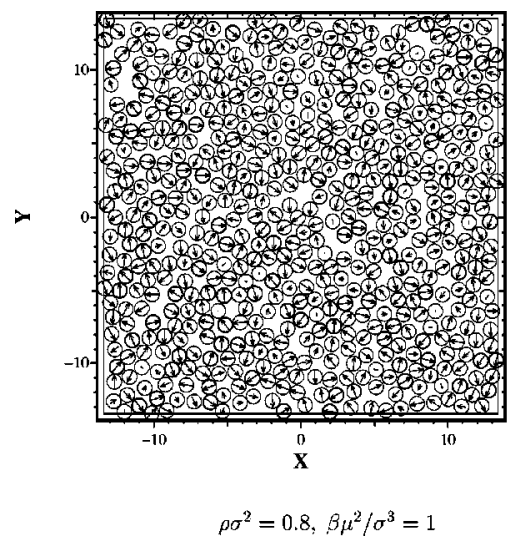

$$
\rho \sigma^{2}=0.8, \beta \mu^{2} / \sigma^{3}=1
$$

FIG. 11. Planar projection of simulation snapshots of a ferrofluid monolayer for two different dipole moments without external field. The arrows indicate the projection of the three dimensional magnetic dipole. 
The correlation functions obtained from the RHNC integral equation are compared with MC simulation data in Figs. 3-8 for various densities and external fields. These states correspond to the paramagnetic fluid regime. This system is known to exhibit a strong tendency to polymerize at low densities and temperatures [26,27], by which in principle one would not expect a gas-liquid transition to occur in the absence of dispersive forces, and hence one cannot properly ascribe these states to a gas or a liquid phase.

It can be appreciated that the optimized RHNC integral equation provides an excellent description of the microscopic structure of the dipolar fluid, with and without external field, except in the case of $\rho \sigma^{2}=0.8$. Here, the integral equation renders a much more pronounced orientational structure while the spatial ordering is exactly reproduced. This discrepancy will be further discussed below when analyzing the thermodynamic properties. The behavior at large separations is well reproduced, including the crossover of $h^{110}(r)$ and $h^{112}(r)$, which is driven by the limiting behavior defined in Eqs. (59) and (60) when the symmetry with respect to the plane is broken by the external field. It can also be seen that the contact values of the correlation functions decrease slightly as the field is augmented; this is due to the fact that the out-of-plane alignment induced by the field introduces repulsive dipole-dipole interactions. This effect is obviously less significant at low densities.

Finally, we collect in Table I all the thermodynamic and magnetic properties calculated in RHNC approximation. Those properties for which MC and DFT data are also available are compared with the RHNC values in Table II and are plotted in Fig. 9. In accord with the results for the structure, the thermodynamic description provided by the RHNC equation is also found to be in good agreement with simulation. Once again, the DFT results are semiquantitative and their quality worsens considerably as the density increases.

It is worth noting that we have encountered convergence problems in the RHNC solutions for reduced densities above 0.8. As can be seen in Table I and Fig. 10, this corresponds to a region where the transverse (i.e., in-plane) susceptibility starts to rise appreciably and is where the orientational order (see Figs. 7 and 8) predicted by the integral equation is more pronounced than what is actually found in the simulation. This orientational structure disagreement is in consonance with the discrepancies in the behavior of the transverse susceptibility depicted in Fig. 10. Thus, whereas the increase in the theoretical susceptibility and the difficulties in convergence seem to indicate that the system might be close to some sort of in-plane order-disorder transition, the simulation predicts no anomalous behavior for these high-density states. Nonetheless, at somewhat higher dipole moment the simulation results start to show a clear head-to-tail in-plane alignment of the dipoles with formation of vortex structures, as can be appreciated in Fig. 11. It thus might happen that the integral equation underestimates the value of the transition dipole moment. Alternatively, it could be the case that the ordering process for a low-dipole moment in a finite system is an extremely slow one that is only captured by the simulation at lower temperatures, although calculations for various systems sizes and lengths of the run do not seem to support this possibility. The situation thus remains inconclusive, though we are of the opinion that the lack of convergence in the integral equation procedure is indicating the onset of some in-plane ordering.

In summary, we have presented an integral equation formalism that is able to provide an accurate description of fluidlike dipolar monolayers for both their one-particle and two-particle structural properties and for their thermodynamics. A simple DFT treatment has been shown to be capable of providing a qualitatively correct picture of the fluid behavior. Future work on this model will focus on the study of possible in-plane transitions, incorporating exchange interactions and magnetic surface anisotropy.

\section{ACKNOWLEDGMENTS}

E.L. thanks the Dirección General de Enseñanza Superior e Investigación Científica (Spain) for partial support of this work under Grant No. PB97-0258-C02-02. Computing time on a CRAY C-98 was granted by the Institut de Développement et de Ressources en Informatique (IDRIS). The Laboratoire de Physique Théorique is Unité Mixte de Recherche No. 8624 du Center National de Recherche Scientifique.
[1] M. B. Taylor and B. L. Gyorffy, J. Phys.: Condens. Matter 5, 4527 (1993).

[2] J. G. Gay and R. Richter, Phys. Rev. Lett. 56, 2728 (1986).

[3] K. Zahn, J. M. Méndez-Alcaraz, and G. Maret, Phys. Rev. Lett. 79, 175 (1997).

[4] K. Zahn, R. Lenke, and G. Maret, Phys. Rev. Lett. 82, 2721 (1999).

[5] A. P. Gast and W. B. Russel, Phys. Today 51, 24 (1998), and references therein.

[6] D. Henderson, in Fundamentals of Inhomogeneous Fluids, edited by D. Henderson (Dekker, New York, 1992), Chap. 4.

[7] F. Lado and E. Lomba, Phys. Rev. Lett. 80, 3535 (1998).

[8] F. Lado, E. Lomba, and J. J. Weis, Phys. Rev. E 58, 3478 (1998); 60, 2429 (1999).

[9] F. Lado, Phys. Rev. E 55, 426 (1997).

[10] F. Lado, E. Lomba, and M. Lombardero, J. Chem. Phys. 108, 4530 (1998).
[11] F. Lado, Phys. Rev. E 54, 4411 (1996).

[12] F. Lado, J. Chem. Phys. 108, 6441 (1998).

[13] B. Groh and S. Dietrich, Phys. Rev. E 50, 3814 (1994); 53, 2509 (1996).

[14] P. I. Teixeira and M. M. Telo da Gama, J. Phys.: Condens. Matter 3, 111 (1991).

[15] J. J. Weis, M. J. P. Nijmeijer, J. M. Tavares, and M. M. Telo da Gama, Phys. Rev. E 55, 436 (1997).

[16] J. P. Hansen and I. R. McDonald, Theory of Simple Liquids (Academic, London, 1986).

[17] F. Lado, Phys. Rev. A 8, 2548 (1973).

[18] Y. Rosenfeld and N. W. Ashcroft, Phys. Rev. A 20, 1208 (1979).

[19] F. Lado, Phys. Lett. A 89A, 196 (1982).

[20] W. H. Press and S. A. Teukolsky, Comput. Phys. 4, 423 (1990).

[21] F. Lado, J. Chem. Phys. 49, 3092 (1968). 
[22] F. Lado, J. Comput. Phys. 8, 417 (1971).

[23] F. Lado, Mol. Phys. 47, 283 (1982).

[24] See routine BCPOL from the IMSL Mathematical Library.

[25] G. T. Gao, X. C. Zeng, and W. Wang, J. Chem. Phys. 106,
3311 (1997).

[26] J. J. Weis, Mol. Phys. 93, 361 (1998).

[27] S. W. Davis, W. McCausland, H. C. McGahagan, C. T. Tanaka, and M. Widom, Phys. Rev. E 59, 2424 (1999). 